\title{
Effect of Educational Program on Maternity Nurses' Knowledge About Antepartum Hemorrhage at Women's Health Hospital, Assiut University
}

\author{
Engy S. Gad-EIrab ${ }^{1}$, Walaa H. Ibrahim ${ }^{2}$, Hamida A-E. Abd El-Hafize ${ }^{3}$ \& Ahmed N. Hassan ${ }^{4}$. \\ ${ }^{1 .}$ Supervisor Nurse, Women Health Hospital, Assiut University, Egypt. \\ ${ }^{2 .}$ Lecturer of Maternity \& Newborn Health Nursing, Faculty of Nursing, Assiut University, Egypt. \\ ${ }^{33}$ Assistant Professor of Maternity \& Newborn Health Nursing, Faculty of Nursing, Assiut University, Egypt. \\ ${ }^{4 .}$ Assistant Professor of Obstetrics \& Gynecological Medicine, Faculty of Medicin, Assiut University, Egypt.
}

\begin{abstract}
Background: Ante partum hemorrhage (APH) is a grave obstetrical emergency and is a leading cause of maternal and perinatal mortality and morbidity, and nurses play a major role in the care of women with APH. Aim: assess maternity nurses' knowledge about APH, and evaluate effect of educational program on improving maternity nurses' knowledge regarding APH. Methods: Quasi experimental research design was used in this study. It was conducted at Women Health Hospital, Assiut University in places that deal with APH patients. Sample included 86 maternity nurses. Structured interview questionnaire was used and included two parts. An educational program was implemented to maternity nurses on APH. Results: there is highly statistical significance difference between pre \&posttest regarding total knowledge of studied maternity nurses regarding APH p-value 0.001. Conclusion: there was an improvement in the knowledge of maternity nurses regarding APH as evidenced by the pretest and posttest knowledge scores. Recommendations: provide a continuous education and training program to maternity nursing staff on APH.
\end{abstract}

\section{Keywords: Antepartum Hemorrhage, Educational Program, Knowledge \& Maternity Nurses.}

\section{Introduction}

Ante partum hemorrhage (APH) is a grave obstetrical emergency and is a main cause of maternal and perinatal mortality and morbidity. APH is any bleeding from or into the genital tract after the period of viability (20weeks of gestation) and before the end of second stage of labour. There are two main types of Ante partum hemorrhage placenta previa and abruption placenta. Partially or completely implanted placenta in the lower uterine segment is called placenta previa. Advancing maternal age, multiparity, prior cesarean section, and prior abortions are

Incidence of placenta previa in the developed countries is between $0.29 \%$ and $1.24 \%$. Pregnancy that complicated by placenta previa have a higher rates of second trimester bleeding, abnormal presentations of fetus, congenital malformations, cesarean delivery, postpartum hemorrhage, prematurity... etc., compared to pregnancy without placenta previa (Omokanye et al., 2017).

Placenta previa has a four grades, ranging from minor to major. The first one is low-lying placenta in which placenta lies in the lower uterine segment but its lower edge does not abut the internal cervical os. And this type has a good chance of having a vaginal delivery. The second is marginal previa in which placental tissue reaches the margin of the internal cervical os, but does not cover it, vaginal birth is still possible. Third is partial previa in which the placenta covers part of the cervix and the forth is major or complete, this is the most serious type. In major placenta previa, the placenta will eventually cover the entire cervix entire cervix that increases risk for APH, cesarean section is usually recommended, and increased probability of premature baby in severe cases (Debra Sullivan, 2016).

Placental abruption is the premature separation of the implanted placenta before the delivery of the fetus (Ananth et al., 2016). Advanced maternal age, high parity, fertility treatments, second trimester bleeding and premature rupture of membranes are significant risk factors for placental abruption (Okby et al., 2017). Placental abruption usually accompanied with bleeding, contraction of the uterus, and fetal distress. It is a main cause of third-trimester bleeding that associated with maternal and fetal morbidity and mortality. Placental abruption is classified based on extent of separation as partial or complete and location of separation as marginal or central (Rao \& Hari, 2016).

The maternal complications in patients with APH are mal presentation, premature labour, postpartum hemorrhage, sepsis, shock and retained placenta. Various fetal complications are premature baby, low birth weight, intrauterine death, congenital malformation and birth asphyxia (Bishnoi, 2013). 
Nurses play a major role in the care of a woman with APH. They are also entrusted with the outcome of the lives of both the mother and the child. Nurse should assess baseline vital signs especially the blood pressure. The physician would order monitoring of the blood pressure every 5-15 minutes. Nurse should assess fetal heart sounds to monitor the wellbeing of the fetus ,monitor uterine contractions to establish the progress of labor of the mother, Weigh perineal pads used during bleeding to estimate amount of blood lost and help the woman in a side lying position when bleeding occurs (Marianne, 2017).

A vital role is also upheld by the nurses through accurate assessment would be one of the baseline data for all health care providers to plot the care plan for pregnant woman with APH. Assess for signs of shock, especially when heavy bleeding occurs. Nurse should assess if the bleeding is external or internal, monitor contractions if separation occurs during labor ,Obtain baseline vital signs, assess for the time the bleeding began, the amount and kind of bleeding, and interventions done when bleeding occurred if it started before admission, assess for the quality of pain (Norwitz \& Shin, 2018).

\section{Significance of the study}

Nurses play important role in early detection of high risk factors of APH, in addition, maternity nurses play important role to ensure the safety of the mother and her baby during all phases of pregnancy and delivery. They must have a knowledge about complications that can occur during pregnancy and how to deal with it (Sharon \& Emily 2017).

Ante partum hemorrhage affect $3.8 \%$ globally. Abruptio placenta (56\%) constituted the largest group. Maximum number of patients was in the age group 20 to 30 years in both abruption $(53.5 \%)$ and placenta previa (52.5\%). In abruption $53.6 \%$ and in placenta previa $79 \%$ of the patients were multiparous. Majority (56\%) of the patients with APH had gestational age of 28 to 34 weeks (Jharaik et al., 2019). Prevalence was highest among Asian studies (12.2 per 1000) and lower among studies from Europe (3.6 per 1000), North America (2.9 per 1000) and Sub-Saharan Africa (2.7 per 1000) (Cresswell et al., 2013). In Egypt, the incidence of placenta previa is $(0.3-0.5 \%)$ of the pregnancies, it accounts for about $(20 \%)$ of all cases of antepartum hemorrhage (Fan, et al., 2017).

Aim of the study

This study aimed to

- Assess maternity nurses' knowledge about APH.

- Evaluate the effect of educational program on improving maternity nurses' knowledge regarding APH.
Research hypothesis

H0: No improvement in nurses' knowledge regarding APH after implementation of educational program.

H1: There was an improvement in nurses' knowledge regarding APH after implementation of educational program.

\section{Subjects \& Methods}

Subjects and methods of this study are displayed into four designs technical, operational, administrative, and statistical design.

Technical Design

Which involved research design, setting, study sample, and tools of data collection.

\section{Research Design}

A quasi-experimental (pre-posttest) design was used in this study.

Setting

This study was conducted at Women Health Hospital, Assiut University in places that deal with APH patients as (reception ward that has 14 beds, fetal monitoring and emergency which has 28 bed, inpatient ward which has 98 bed and intensive care ward which has 12 bed). Women Health Hospital service all areas of Upper Egypt. It is a building that have a six floor, five for governmental admission and the last one for private service.

Sample

A total of 86 maternity nurse included in the study, who were close contact with APH patients and involved ( 8 nurses from reception ward, 45 nurses from fetal monitoring and emergency ward, 12 nurses from inpatient ward and 21 nurses from intensive care unit).

Tools of the study

A structured interview questionnaire was developed by the researchers. It involved two parts.

Part one: included personal data of maternity nurses as (age, education, years of experience and attendance training program before).

Part two: included 21 questions to assess maternity nurse knowledge about APH and its care. And involved four section

Section 1: included 5 questions to assess maternity nurses' knowledge about APH as definition, types and complications, 2 of them had more than one answer.

Section 2: included 6 questions to assess maternity nurses' knowledge about placenta previa as definition, types, causes and sings \&symptoms, 3 of them had more than one answer.

Section 3: included 5 questions to assess maternity nurses' knowledge about abruptio placenta as definition, types, causes and sings \&symptoms, 3 of them had more than one answer. 
Section 4: included 5 questions to assess maternity nurses' knowledge about care of APH as Cannula should remain in normal condition for 24 hours, prohibition to use casco examination, rest in bed for stable condition, persistent bleeding should remain in hospital and avoidance of vaginal or anal examination.

\section{Knowledge scoring system}

Each question was scored as (1) for a correct answer and $(0)$ for an incorrect answer. While the total knowledge score was calculated as the following: knowledge was considered inadequate if the percent score was $<75 \%$ and considered adequate if percent was $75 \%$ and more.

\section{Supportive materials}

It was designed by the researcher based on literature review. It prepared in a form of booklet with using a simple and clear Arabic language supported with photo to support some information to the nurses.

\section{Tools Validity}

Tools were reviewed by a panel of 3 experts in the field of maternity and newborn health nursing and obstetrics and gynecological medicine for clarity and comprehensive.

\section{Tools Reliability}

The internal consistency of the tool scale was calculated by using Cronbach's Alpha; and it was 0.841 .

\section{operational design}

It was displayed in two phases piolet study and field work.

Pilot study

Pilot study was included (10\%) according to inclusion of the sample size ( 9 cases) to evaluate the clarity and efficiency of the tool used in the study.

\section{Field work}

Data collection of this study was taken seven months started from the beginning of February 2018, and completed by the end July 2018. This was achieved in three phases, pre intervention, intervention and post intervention:

\section{Pre intervention phase}

Upon securing official permission to conduct the study. The researcher interviewed with each maternity nurse individually, explain the purpose of the study and method of implementation of educational program, and took their consent on participation on the study. After that personal data was taken and ask them to fill data regarding knowledge about APH as a form of pretest. This took a time from 25-35 minutes.

\section{Intervention phase}

Carrying out of educational program which took about 22 weeks or nearly 6 months. The researcher visited the previous involved setting two days/ week. With a performance of two sessions every week to the maternity nurses, each session involved a small group with a number of 2-4 nurse. It was implemented in a form of lecture to each group. Session took a time of 60-90 minute to explain all information regarding APH (definition, types, and complications), placenta previa (definition, types, causes, and sings \&symptoms), abruptio placenta (definition, types, causes, and sings \&symptoms) and care of APH. Different teaching methods was used to reach to intended objective as lecture, group discussion, problem solving and critical thinking. Also a copy of booklet was given to each nurse as a supportive material.

\section{Post intervention phase}

After 4 weeks of the session, the researcher interviewed again with the nurses involved in the session to perform a post test that had the same pretest questions.

\section{Administrative design}

This study was carried out under the approval of faculty of nursing's Ethical committee, Assiut University, also an official permission was obtained from the director of Woman Health Hospital, informed consent was taken from each nurse involved in the study, confidentiality was assured. The nurse was freely to withdraw from the study at any stage.

\section{Statistical design}

The collected data were organized, categorized, coded, tabulated and analyzed using the Statistical Package for social sciences (SPSS). Data were presented and tables and charts using numbers, percentages, means, and standard deviation. Correlation between variables (Pearson correlation) and chi-square test was used statistical significance was considered at $\mathrm{P}$-value $\leq 0.05$. 


\section{Results}

Table (1): Distribution of studied maternity nurses according to personal data.

\begin{tabular}{|l|c|c|}
\hline \multicolumn{1}{|c|}{ Personal data } & No. (n= 86) & $\%$ \\
\hline Age: (years) & 58 & 67.4 \\
\hline Less than 30 year & 28 & 32.6 \\
\hline More than 30 year & \multicolumn{1}{|c|}{$28.45 \pm 2.71$} \\
\hline Age mean I SD & 33 & 38.3 \\
\hline Educational qualification: & 40 & 46.5 \\
\hline Secondary nursing school & 13 & 15.2 \\
\hline Technical institute of nursing & \\
\hline University qualification & 20 & 23.3 \\
\hline Years of experience in obstetrics and gynecology ward: & 20 & 23.3 \\
\hline Less than two years & 18 & 20.8 \\
\hline <2 - 5 years & 28 & 32.6 \\
\hline <5-10 years & & \\
\hline More than 10 years & 35 & 40.7 \\
\hline Attending training courses regarding gynecology and obstetrics & 59.3 \\
\hline Yes & 51 & \\
\hline No & & \\
\hline
\end{tabular}

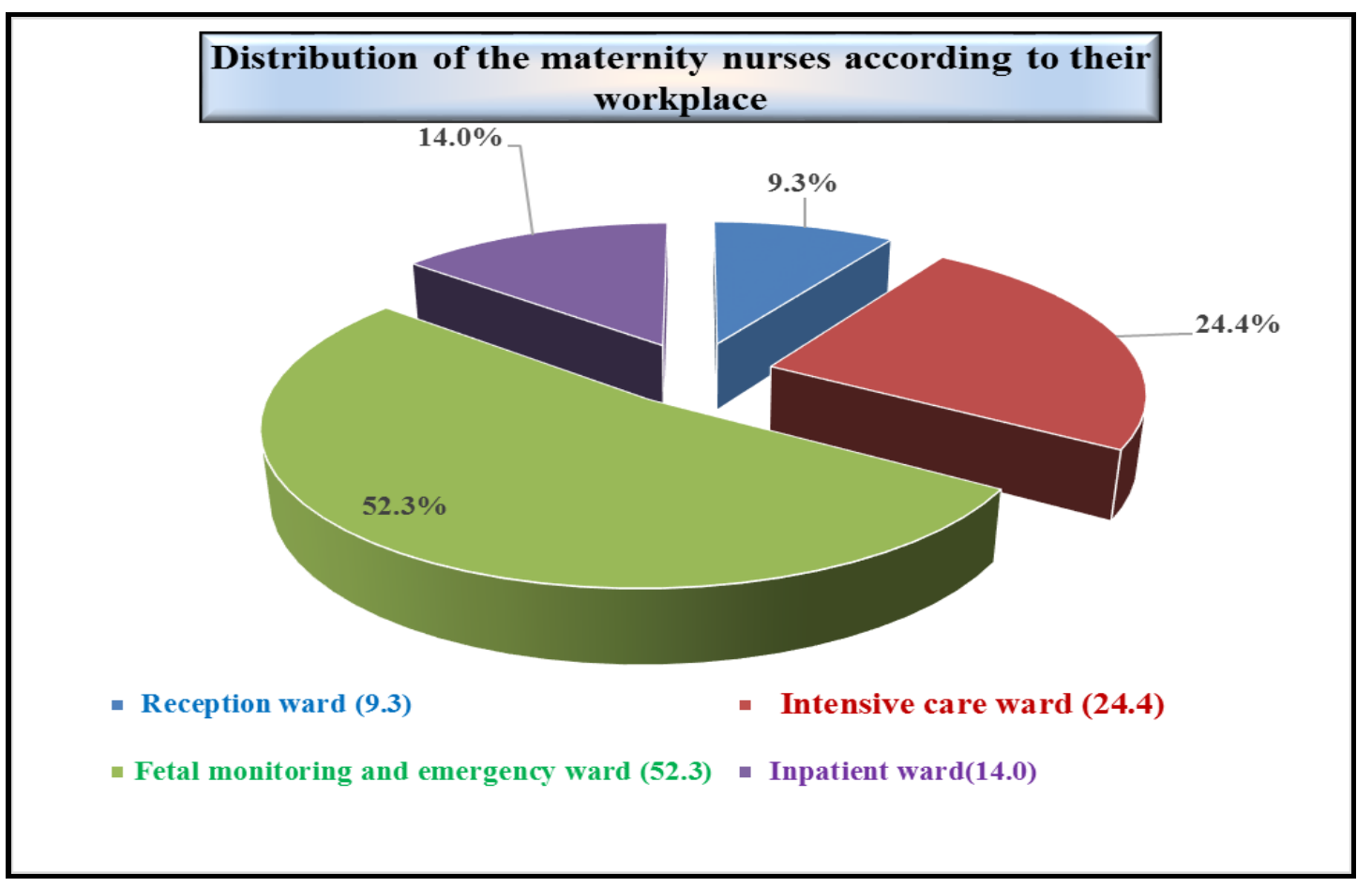

Figure (1): Distribution of studied maternity nurses according to workplace: 
Table (2): Distribution of studied maternity nurses according to general knowledge about antepartum hemorrhage in pre and posttest.

\begin{tabular}{|c|c|c|c|c|c|c|}
\hline \multirow{2}{*}{ Items } & & \multicolumn{2}{|c|}{ Pre-test $(n=86)$} & \multicolumn{2}{|c|}{ Post-test $(n=86)$} & \multirow{2}{*}{ P-value } \\
\hline & & No. & $\%$ & No. & $\%$ & \\
\hline Definition & $\begin{array}{l}\text { Correct } \\
\text { Incorrect }\end{array}$ & $\begin{array}{l}63 \\
23\end{array}$ & $\begin{array}{l}73.3 \\
26.7\end{array}$ & $\begin{array}{c}85 \\
1\end{array}$ & $\begin{array}{c}98.9 \\
1.1\end{array}$ & $0.001 * *$ \\
\hline \multicolumn{7}{|l|}{ Main types of antepartum hemorrhage } \\
\hline Placenta previa & $\begin{array}{l}\text { Correct } \\
\text { Incorrect }\end{array}$ & $\begin{array}{l}54 \\
32\end{array}$ & $\begin{array}{l}62.8 \\
37.2\end{array}$ & $\begin{array}{c}82 \\
4\end{array}$ & $\begin{array}{c}95.3 \\
4.7\end{array}$ & \multirow{2}{*}{$0.001 * *$} \\
\hline Abruptio placenta & $\begin{array}{l}\text { Correct } \\
\text { Incorrect }\end{array}$ & $\begin{array}{l}60 \\
26\end{array}$ & $\begin{array}{l}69.8 \\
30.2\end{array}$ & $\begin{array}{c}81 \\
5\end{array}$ & $\begin{array}{c}94.2 \\
5.8\end{array}$ & \\
\hline \multicolumn{7}{|c|}{ Complications of Antepartum Hemorrhage } \\
\hline \multicolumn{6}{|l|}{ Maternal complications } & \multirow{6}{*}{$0.001 * *$} \\
\hline Hemorrhagic shock & $\begin{array}{l}\text { Correct } \\
\text { Incorrect }\end{array}$ & $\begin{array}{l}58 \\
28\end{array}$ & $\begin{array}{l}67.4 \\
32.6\end{array}$ & $\begin{array}{c}85 \\
1\end{array}$ & $\begin{array}{c}98.9 \\
1.1\end{array}$ & \\
\hline Acute renal failure & $\begin{array}{l}\text { Correct } \\
\text { Incorrect }\end{array}$ & $\begin{array}{l}50 \\
36\end{array}$ & $\begin{array}{l}58.1 \\
41.9\end{array}$ & $\begin{array}{l}71 \\
15\end{array}$ & $\begin{array}{l}82.6 \\
17.4\end{array}$ & \\
\hline $\begin{array}{l}\text { Disseminated intra-vascular coagulation } \\
\text { (DIC) }\end{array}$ & $\begin{array}{l}\text { Correct } \\
\text { Incorrect }\end{array}$ & $\begin{array}{l}33 \\
53\end{array}$ & $\begin{array}{l}38.4 \\
61.6\end{array}$ & $\begin{array}{l}65 \\
21\end{array}$ & $\begin{array}{l}75.6 \\
24.4\end{array}$ & \\
\hline Severe anemia & $\begin{array}{l}\text { Correct } \\
\text { Incorrect }\end{array}$ & $\begin{array}{l}60 \\
26\end{array}$ & $\begin{array}{l}69.8 \\
30.2\end{array}$ & $\begin{array}{c}85 \\
1\end{array}$ & $\begin{array}{c}98.9 \\
1.1\end{array}$ & \\
\hline $\begin{array}{l}\text { Increased risk for postpartum } \\
\text { hemorrhage }\end{array}$ & $\begin{array}{l}\text { Correct } \\
\text { Incorrect }\end{array}$ & $\begin{array}{l}49 \\
37 \\
\end{array}$ & $\begin{array}{l}57.0 \\
43.0 \\
\end{array}$ & $\begin{array}{l}77 \\
9 \\
\end{array}$ & $\begin{array}{l}89.5 \\
10.5 \\
\end{array}$ & \\
\hline \multicolumn{7}{|l|}{ Fetal complications } \\
\hline Prematurity & $\begin{array}{l}\text { Correct } \\
\text { Incorrect }\end{array}$ & $\begin{array}{l}55 \\
31\end{array}$ & $\begin{array}{l}64.0 \\
36.0\end{array}$ & $\begin{array}{c}82 \\
4\end{array}$ & $\begin{array}{c}95.3 \\
4.7\end{array}$ & \multirow{3}{*}{$0.002 * *$} \\
\hline Birth asphyxia & $\begin{array}{l}\text { Correct } \\
\text { Incorrect }\end{array}$ & $\begin{array}{l}60 \\
26\end{array}$ & $\begin{array}{l}69.8 \\
30.2\end{array}$ & $\begin{array}{c}80 \\
6\end{array}$ & $\begin{array}{c}93.0 \\
7.0\end{array}$ & \\
\hline Intrauterine fetal death & $\begin{array}{l}\text { Correct } \\
\text { Incorrect }\end{array}$ & $\begin{array}{l}63 \\
23\end{array}$ & $\begin{array}{l}73.3 \\
26.7\end{array}$ & $\begin{array}{c}78 \\
8\end{array}$ & $\begin{array}{c}90.7 \\
9.3\end{array}$ & \\
\hline
\end{tabular}

Mc Nemar test used for pair qualitative variables

(**) highly statistically significant $p<0.01$ 
Table (3): Distribution of studied maternity nurses according to knowledge about placenta previa in pre and posttest.

\begin{tabular}{|c|c|c|c|c|c|c|}
\hline \multirow{2}{*}{ Items } & & \multicolumn{2}{|c|}{ Pre-test $(n=86)$} & \multicolumn{2}{|c|}{ Post-test $(n=86)$} & \multirow{2}{*}{ P-value } \\
\hline & & No. & $\%$ & No. & $\%$ & \\
\hline \multicolumn{7}{|c|}{ General information } \\
\hline Definition & Correct & 52 & 60.5 & 82 & 95.3 & \multirow{6}{*}{$0.001 * *$} \\
\hline & Incorrect & 34 & 39.5 & 4 & 4.7 & \\
\hline Place of placenta attachment & Correct & 63 & 73.3 & 85 & 98.9 & \\
\hline & Incorrect & 23 & 26.7 & 1 & 1.1 & \\
\hline Placenta previa occurred in the second & Correct & 31 & 36.0 & 75 & 87.2 & \\
\hline trimester of pregnancy & Incorrect & 55 & 64.0 & 11 & 12.8 & \\
\hline \multicolumn{7}{|l|}{ Degree of placenta previa } \\
\hline Low lying & Correct & 43 & 50.0 & 82 & 95.3 & \multirow{8}{*}{$0.001 * *$} \\
\hline & Incorrect & 43 & 50.0 & 4 & 4.7 & \\
\hline Marginal & Correct & 52 & 60.5 & 81 & 94.2 & \\
\hline & Incorrect & 34 & 39.5 & 5 & 5.8 & \\
\hline Partial & Correct & 55 & 64.0 & 80 & 93.0 & \\
\hline & Incorrect & 31 & 36.0 & 6 & 7.0 & \\
\hline Complete & Correct & 62 & 72.1 & 83 & 96.5 & \\
\hline & Incorrect & 24 & 27.9 & 3 & 3.5 & \\
\hline \multicolumn{7}{|c|}{ Causes and risk factors of placenta previa: } \\
\hline \multirow[t]{2}{*}{ Age of $<18$ or $<35$ vyears } & Correct & 32 & 37.2 & 75 & 87.2 & \multirow{10}{*}{$0.001 * *$} \\
\hline & Incorrect & 54 & 62.8 & 11 & 12.8 & \\
\hline \multirow[t]{2}{*}{ More than one cesarean section } & Correct & 40 & 46.5 & 71 & 82.6 & \\
\hline & Incorrect & 46 & 53.5 & 15 & 17.4 & \\
\hline \multirow[t]{2}{*}{ Less space between children } & Correct & 29 & 33.7 & 69 & 80.2 & \\
\hline & Incorrect & 57 & 66.3 & 17 & 19.8 & \\
\hline \multirow[t]{2}{*}{ Previous placenta previa } & Correct & 60 & 69.8 & 85 & 98.9 & \\
\hline & Incorrect & 26 & 30.2 & 1 & 1.1 & \\
\hline \multirow{2}{*}{ Large placenta size } & Correct & 25 & 29.1 & 68 & 79.1 & \\
\hline & Incorrect & 61 & 70.9 & 18 & 20.9 & \\
\hline \multicolumn{7}{|c|}{ Signs and symptom of placenta previa } \\
\hline \multirow{2}{*}{$\begin{array}{l}\text { Vaginal bleeding, causeless, painless } \\
\text { except during labor, and recurrent }\end{array}$} & Correct & 62 & 72.1 & 86 & 100.0 & \multirow{12}{*}{$0.001 * *$} \\
\hline & Incorrect & 24 & 27.9 & 0 & 0.0 & \\
\hline \multirow{2}{*}{$\begin{array}{l}\text { Usually vaginal bleeding occurred during } \\
\text { rest or sleep }\end{array}$} & Correct & 44 & 51.2 & 79 & 91.9 & \\
\hline & Incorrect & 42 & 48.8 & 7 & 8.1 & \\
\hline \multirow{2}{*}{$\begin{array}{l}\text { Fresh blood color that usually stop } \\
\text { suddenly }\end{array}$} & Correct & 60 & 69.8 & 75 & 87.2 & \\
\hline & Incorrect & 26 & 30.2 & 11 & 12.8 & \\
\hline \multirow[t]{2}{*}{ The uterus is lax and not tender } & Correct & 58 & 67.4 & 68 & 79.1 & \\
\hline & Incorrect & 28 & 32.6 & 18 & 20.9 & \\
\hline \multirow[t]{2}{*}{ Mal-presentation is common } & Correct & 68 & 79.1 & 75 & 87.2 & \\
\hline & Incorrect & 18 & 20.9 & 11 & 12.8 & \\
\hline \multirow[t]{2}{*}{ Presenting part is not engaged } & Correct & 48 & 55.8 & 78 & 90.7 & \\
\hline & Incorrect & 38 & 44.2 & 8 & 9.3 & \\
\hline
\end{tabular}

Mc Nemar test used for pair qualitative variables

(**) highly statistically significant $p<0.01$ 
Table (4): Distribution of studied maternity nurses according to knowledge about abruptio placenta in pre and posttest.

\begin{tabular}{|c|c|c|c|c|c|c|}
\hline \multirow[t]{2}{*}{ Items } & & \multicolumn{2}{|c|}{$\begin{array}{c}\text { Pre-test } \\
(n=86)\end{array}$} & \multicolumn{2}{|c|}{$\begin{array}{c}\text { Post-test } \\
(n=86)\end{array}$} & \multirow[t]{2}{*}{ P-value } \\
\hline & & No. & $\%$ & No. & $\%$ & \\
\hline \multicolumn{7}{|c|}{ General information } \\
\hline Definition & \begin{tabular}{|l|} 
Correct \\
Incorrect
\end{tabular} & $\begin{array}{l}68 \\
18\end{array}$ & $\begin{array}{l}79.1 \\
20.9\end{array}$ & $\begin{array}{c}79 \\
7\end{array}$ & $\begin{array}{c}91.9 \\
8.1\end{array}$ & \multirow[b]{2}{*}{$0.002 * *$} \\
\hline Place of placenta attachment & \begin{tabular}{|l|} 
Correct \\
Incorrect \\
\end{tabular} & $\begin{array}{l}63 \\
23 \\
\end{array}$ & $\begin{array}{l}73.3 \\
26.8 \\
\end{array}$ & $\begin{array}{c}85 \\
1 \\
\end{array}$ & $\begin{array}{c}98.9 \\
1.1\end{array}$ & \\
\hline \multicolumn{7}{|l|}{ Types of abruptio placenta } \\
\hline Revealed & $\begin{array}{l}\text { Correct } \\
\text { Incorrect } \\
\end{array}$ & $\begin{array}{l}55 \\
31 \\
\end{array}$ & $\begin{array}{l}64.0 \\
36.0 \\
\end{array}$ & $\begin{array}{c}84 \\
2 \\
\end{array}$ & $\begin{array}{c}97.7 \\
2.3 \\
\end{array}$ & \multirow{3}{*}{$0.001 * *$} \\
\hline Concealed & \begin{tabular}{|l|} 
Correct \\
Incorrect \\
\end{tabular} & $\begin{array}{l}40 \\
46 \\
\end{array}$ & $\begin{array}{l}46.5 \\
53.5 \\
\end{array}$ & $\begin{array}{c}85 \\
1 \\
\end{array}$ & $\begin{array}{c}98.9 \\
1.1 \\
\end{array}$ & \\
\hline Combined & $\begin{array}{l}\text { Correct } \\
\text { Incorrect }\end{array}$ & $\begin{array}{l}41 \\
45\end{array}$ & $\begin{array}{l}47.7 \\
52.3\end{array}$ & $\begin{array}{c}82 \\
4\end{array}$ & $\begin{array}{c}95.3 \\
4.7\end{array}$ & \\
\hline \multicolumn{7}{|c|}{ Causes and risk factors of abruptio placenta: } \\
\hline Pregnancy induced hypertension $(\mathrm{PIH})$ & $\begin{array}{l}\text { Correct } \\
\text { Incorrect }\end{array}$ & $\begin{array}{l}56 \\
30\end{array}$ & $\begin{array}{l}65.1 \\
34.9\end{array}$ & $\begin{array}{c}81 \\
5\end{array}$ & $\begin{array}{c}94.2 \\
5.8\end{array}$ & \multirow{5}{*}{$0.001 * *$} \\
\hline Trauma & $\begin{array}{l}\text { Correct } \\
\text { Incorrect } \\
\end{array}$ & $\begin{array}{l}60 \\
26 \\
\end{array}$ & $\begin{array}{l}69.8 \\
30.2 \\
\end{array}$ & $\begin{array}{c}80 \\
6 \\
\end{array}$ & $\begin{array}{c}93.0 \\
7.0 \\
\end{array}$ & \\
\hline Deficiencies in vitamins $\mathrm{C}$ and $\mathrm{K}$ & $\begin{array}{l}\text { Correct } \\
\text { Incorrect }\end{array}$ & $\begin{array}{l}56 \\
30\end{array}$ & $\begin{array}{l}65.1 \\
34.9\end{array}$ & $\begin{array}{c}81 \\
5\end{array}$ & $\begin{array}{c}94.2 \\
5.8\end{array}$ & \\
\hline Traction on a short umbilical cord & $\begin{array}{l}\text { Correct } \\
\text { Incorrect }\end{array}$ & $\begin{array}{l}36 \\
50\end{array}$ & $\begin{array}{l}41.9 \\
58.1\end{array}$ & $\begin{array}{c}79 \\
7\end{array}$ & $\begin{array}{c}91.9 \\
8.1\end{array}$ & \\
\hline Sudden reduction of the size of the uterus & $\begin{array}{l}\text { Correct } \\
\text { Incorrect } \\
\end{array}$ & $\begin{array}{l}31 \\
55 \\
\end{array}$ & $\begin{array}{l}36.0 \\
64.0 \\
\end{array}$ & $\begin{array}{c}77 \\
9 \\
\end{array}$ & $\begin{array}{l}89.5 \\
10.5 \\
\end{array}$ & \\
\hline \multicolumn{7}{|c|}{ Signs and symptom of abruptio placenta } \\
\hline \multicolumn{7}{|l|}{ For revealed } \\
\hline Vaginal bleeding & $\begin{array}{l}\text { Correct } \\
\text { Incorrect }\end{array}$ & $\begin{array}{l}62 \\
24 \\
\end{array}$ & $\begin{array}{l}72.1 \\
27.9 \\
\end{array}$ & $\begin{array}{c}86 \\
0 \\
\end{array}$ & $\begin{array}{c}100.0 \\
0.0 \\
\end{array}$ & \multirow{5}{*}{$0.001 * *$} \\
\hline $\begin{array}{l}\text { Signs of blood loss are present as (pale, } \\
\text { irritable, increased pulse). }\end{array}$ & $\begin{array}{l}\text { Correct } \\
\text { Incorrect } \\
\end{array}$ & $\begin{array}{l}56 \\
30 \\
\end{array}$ & $\begin{array}{l}65.1 \\
34.9 \\
\end{array}$ & $\begin{array}{l}75 \\
11 \\
\end{array}$ & $\begin{array}{l}87.2 \\
12.8 \\
\end{array}$ & \\
\hline Laxed uterus between contractions & $\begin{array}{l}\text { Correct } \\
\text { Incorrect }\end{array}$ & $\begin{array}{l}44 \\
42\end{array}$ & $\begin{array}{l}51.2 \\
48.8\end{array}$ & $\begin{array}{l}72 \\
14\end{array}$ & $\begin{array}{l}83.7 \\
16.3\end{array}$ & \\
\hline Fetal parts are easily felt & $\begin{array}{l}\text { Correct } \\
\text { Incorrect }\end{array}$ & $\begin{array}{l}58 \\
28\end{array}$ & $\begin{array}{l}67.4 \\
32.6\end{array}$ & $\begin{array}{l}68 \\
18\end{array}$ & $\begin{array}{l}79.1 \\
20.9\end{array}$ & \\
\hline $\begin{array}{l}\text { FHS are heard if less than half of the } \\
\text { placenta is separated. }\end{array}$ & $\begin{array}{l}\text { Correct } \\
\text { Incorrect }\end{array}$ & $\begin{array}{l}68 \\
18 \\
\end{array}$ & $\begin{array}{l}79.1 \\
20.9 \\
\end{array}$ & $\begin{array}{l}75 \\
11 \\
\end{array}$ & $\begin{array}{l}87.2 \\
12.8 \\
\end{array}$ & \\
\hline \multicolumn{7}{|l|}{ For Concealed } \\
\hline $\begin{array}{l}\text { Sudden, severe abdominal pain followed } \\
\text { by fainting and vomiting }\end{array}$ & \begin{tabular}{|l|} 
Correct \\
Incorrect \\
\end{tabular} & $\begin{array}{l}52 \\
34 \\
\end{array}$ & $\begin{array}{l}60.5 \\
39.5 \\
\end{array}$ & $\begin{array}{l}74 \\
12 \\
\end{array}$ & $\begin{array}{l}86.0 \\
14.0 \\
\end{array}$ & \multirow{4}{*}{$0.001 * *$} \\
\hline $\begin{array}{l}\text { Patient becomes pale and irritable, shock } \\
\text { is always present }\end{array}$ & $\begin{array}{l}\text { Correct } \\
\text { Incorrect }\end{array}$ & $\begin{array}{l}34 \\
52\end{array}$ & $\begin{array}{l}39.5 \\
60.5\end{array}$ & $\begin{array}{c}78 \\
8\end{array}$ & $\begin{array}{c}90.7 \\
9.3\end{array}$ & \\
\hline $\begin{array}{l}\text { The uterus is very hard and larger than } \\
\text { expected }\end{array}$ & $\begin{array}{l}\text { Correct } \\
\text { Incorrect }\end{array}$ & $\begin{array}{l}51 \\
35\end{array}$ & $\begin{array}{l}59.3 \\
40.7\end{array}$ & $\begin{array}{l}69 \\
17\end{array}$ & $\begin{array}{l}80.2 \\
19.8\end{array}$ & \\
\hline $\begin{array}{l}\text { Difficulty to feel fetal parts and to hear } \\
\text { FHS }\end{array}$ & $\begin{array}{l}\text { Correct } \\
\text { Incorrect }\end{array}$ & $\begin{array}{l}55 \\
31\end{array}$ & $\begin{array}{l}64.0 \\
36.0\end{array}$ & $\begin{array}{c}80 \\
6\end{array}$ & $\begin{array}{c}93.0 \\
7.0\end{array}$ & \\
\hline
\end{tabular}

Mc Nemar test used for pair qualitative variables

(**) highly statistically significant $p<0.01$ 
Table (5): Distribution of studied maternity nurses according to knowledge about nursing care of patient with APH in pre and posttest:

\begin{tabular}{|c|c|c|c|c|c|}
\hline \multirow[t]{2}{*}{ Item } & \multicolumn{2}{|c|}{$\begin{array}{c}\text { Pre-test } \\
(n=86)\end{array}$} & \multicolumn{2}{|c|}{$\begin{array}{c}\text { Post-test } \\
(n=86)\end{array}$} & \multirow[t]{2}{*}{ P-value } \\
\hline & No. & $\%$ & No. & $\%$ & \\
\hline \multicolumn{5}{|l|}{$\begin{array}{l}\text { Cannula should remain in normal condition for } 24 \text { hours } \\
\text { and be discussed with the medical obstetric team before } \\
\text { removing it: }\end{array}$} & \multirow{3}{*}{$0.001 * *$} \\
\hline Correct & 67 & 77.9 & 82 & 95.3 & \\
\hline Incorrect & 19 & 22.1 & 4 & 4.7 & \\
\hline \multicolumn{5}{|l|}{$\begin{array}{l}\text { It is prohibited to use a casco examination to evaluate } \\
\text { vaginal bleeding: }\end{array}$} & \multirow{3}{*}{$0.001 * *$} \\
\hline Correct & 17 & 19.8 & 85 & 98.9 & \\
\hline Incorrect & 69 & 80.2 & 1 & 1.1 & \\
\hline \multicolumn{5}{|l|}{$\begin{array}{l}\text { Patients who are in stable condition: They are advised to } \\
\text { rest in bed while only allowing going to the toilet so that } \\
\text { there is no bleeding attack for } 24 \text { hours: }\end{array}$} & \multirow[t]{3}{*}{$\mathbf{0 . 0 3 3}^{*}$} \\
\hline Correct & 68 & 79.1 & 78 & 90.7 & \\
\hline Incorrect & 18 & 20.9 & 8 & 9.3 & \\
\hline \multicolumn{5}{|l|}{$\begin{array}{l}\text { Women who suffer from persistent bleeding should remain } \\
\text { in the hospital: }\end{array}$} & \multirow{3}{*}{$0.001 * *$} \\
\hline Correct & 73 & 84.9 & 85 & 98.9 & \\
\hline Incorrect & 13 & 15.1 & 1 & 1.1 & \\
\hline \multicolumn{5}{|l|}{$\begin{array}{l}\text { No vaginal or anal examination may be performed on ante } \\
\text { partum hemorrhage patient: }\end{array}$} & \multirow{3}{*}{$0.001 * *$} \\
\hline Correct & 61 & 70.9 & 78 & 90.7 & \\
\hline Incorrect & 25 & 29.1 & 8 & 9.3 & \\
\hline
\end{tabular}

Mc Nemar test used for pair qualitative variables

(**) highly statistically significant $p<0.01$

(*) statistically significant $p<0.05$

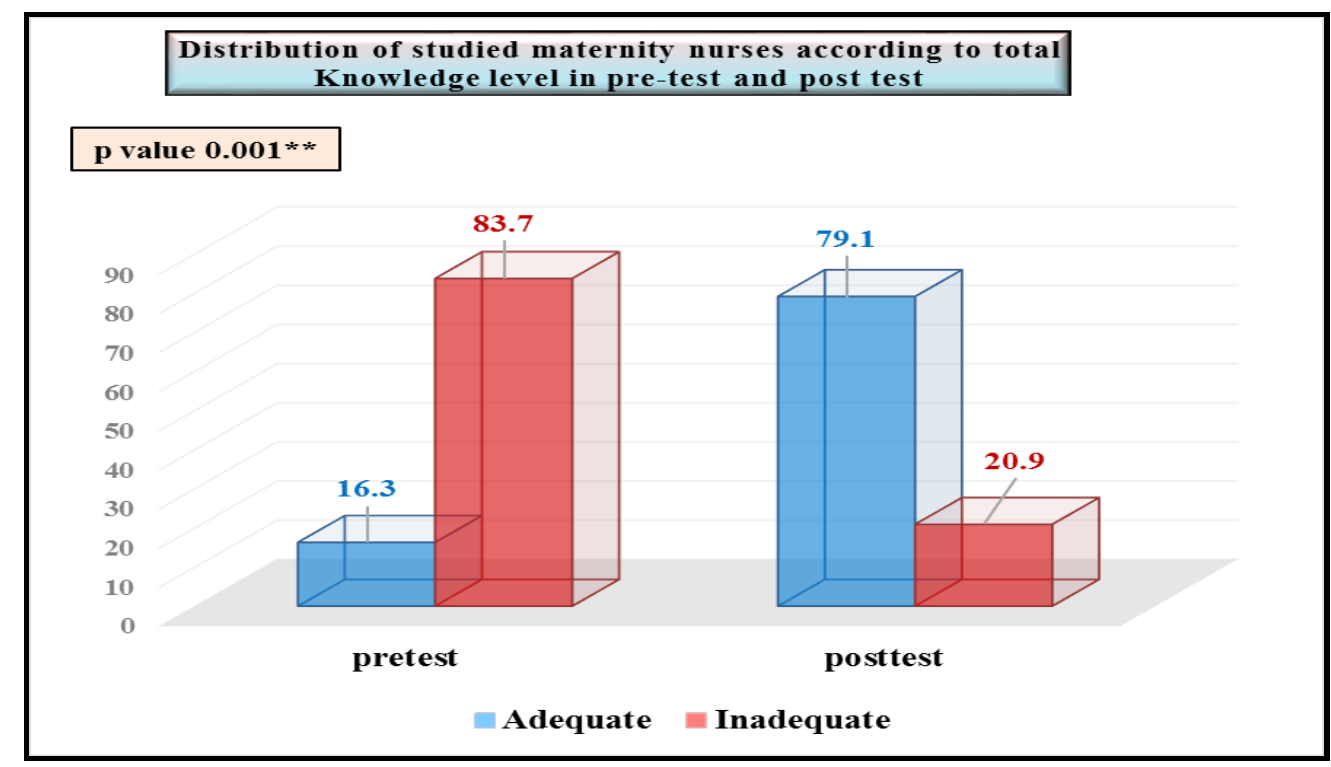

Figure (2): Distribution of studied maternity nurses according to total knowledge in pre-test and posttest. 
Table (6): Distribution of studied maternity nurses according to relation between total knowledge in pretest and personal data.

\begin{tabular}{|c|c|c|c|c|c|c|}
\hline \multirow{3}{*}{ Personal data } & \multicolumn{4}{|c|}{ Total Knowledge } & \multirow{3}{*}{$\begin{array}{c}\text { Total } \\
\text { No }(\%)\end{array}$} & \multirow{3}{*}{ P-value } \\
\hline & \multicolumn{2}{|c|}{ Adequate $(n=14)$} & \multicolumn{2}{|c|}{ Inadequate $(n=72)$} & & \\
\hline & No. & $\%$ & No. & $\%$ & & \\
\hline \multicolumn{6}{|l|}{ Age: (years) } & \multirow{3}{*}{$0.003 * *$} \\
\hline Less than 30 year & 5 & 8.6 & 53 & 91.4 & $58(100.0)$ & \\
\hline More than 30 year & 9 & 32.1 & 19 & 67.9 & $28(100.0)$ & \\
\hline \multicolumn{6}{|l|}{ Workplace: } & \multirow{5}{*}{0.199} \\
\hline Reception ward & 2 & 25.0 & 6 & 75.0 & $8(100.0)$ & \\
\hline Intensive care ward & 2 & 9.5 & 19 & 90.5 & $21(100.0)$ & \\
\hline Inpatient ward & 0 & 0.0 & 12 & 100.0 & $12(100.0)$ & \\
\hline Fetal monitoring and emergency ward & 10 & 22.2 & 35 & 77.8 & $45(100.0)$ & \\
\hline \multicolumn{6}{|l|}{ Educational qualification: } & \multirow{4}{*}{$0.001 * *$} \\
\hline Secondary nursing school & 1 & 3.0 & 32 & 97.0 & $33(100.0)$ & \\
\hline Technical institute of nursing & 3 & 7.5 & 37 & 92.5 & $40(100.0)$ & \\
\hline University qualification & 10 & 76.9 & 3 & 23.1 & $13(100.0)$ & \\
\hline \multicolumn{6}{|c|}{ Years of experience in obstetrics and gynecology } & \multirow{5}{*}{$0.002 * *$} \\
\hline Less than two years & 1 & 5.0 & 19 & 95.0 & $20(100.0)$ & \\
\hline$<2-5$ years & 1 & 5.0 & 19 & 95.0 & $20(100.0)$ & \\
\hline$<5-10$ years & 4 & 22.2 & 14 & 77.8 & $18(100.0)$ & \\
\hline More than 10 years & 8 & 28.6 & 20 & 71.4 & $28(100.0)$ & \\
\hline \multicolumn{6}{|c|}{ Attending training courses regarding gynecology and obstetrics } & \multirow{3}{*}{0.678} \\
\hline Yes & 5 & 14.3 & 30 & 85.7 & $35(100.0)$ & \\
\hline No & 9 & 17.6 & 42 & 82.4 & $51(100.0)$ & \\
\hline
\end{tabular}

(**) highly statistically significant $p<0.01$

Table (7): Distribution of studied maternity nurses according to relation between total knowledge in posttest and personal data.

\begin{tabular}{|c|c|c|c|c|c|c|}
\hline \multirow{3}{*}{ personal data } & \multicolumn{4}{|c|}{ Total Knowledge } & \multirow{3}{*}{$\begin{array}{c}\text { Total } \\
\text { No }(\%) \\
\end{array}$} & \multirow{3}{*}{ P-value } \\
\hline & \multicolumn{2}{|c|}{ Adequate $(n=68)$} & \multicolumn{2}{|c|}{ Inadequate $(n=18)$} & & \\
\hline & No. & $\%$ & No. & $\%$ & & \\
\hline \multicolumn{6}{|l|}{ Age: (years) } & \multirow{3}{*}{$0.005 * *$} \\
\hline Less than 30 year & 43 & 74.1 & 15 & 25.9 & $58(100.0)$ & \\
\hline More than 30 year & 25 & 89.3 & 3 & 10.7 & $28(100.0)$ & \\
\hline \multicolumn{6}{|l|}{ Workplace } & \multirow{5}{*}{0.495} \\
\hline Reception ward & 6 & 75.0 & 2 & 25.0 & $8(100.0)$ & \\
\hline Intensive care ward & 17 & 80.9 & 4 & 19.1 & $21(100.0)$ & \\
\hline Inpatient ward & 10 & 83.3 & 2 & 16.7 & $12(100.0)$ & \\
\hline Fetal monitoring and emergency ward & 35 & 77.8 & 10 & 22.2 & $45(100.0)$ & \\
\hline \multicolumn{6}{|l|}{ Educational qualification } & \multirow{4}{*}{$0.001 * *$} \\
\hline Secondary nursing school & 18 & 54.5 & 15 & 45.5 & $33(100.0)$ & \\
\hline Technical institute of nursing & 38 & 95.0 & 2 & 5.0 & $40(100.0)$ & \\
\hline University qualification & 12 & 92.3 & 1 & 7.7 & $13(100.0)$ & \\
\hline \multicolumn{6}{|c|}{ Years of experience in obstetrics and gynecology } & \multirow{5}{*}{$\mathbf{0 . 0 0 3} * *$} \\
\hline Less than two years & 12 & 60.0 & 8 & 40.0 & $20(100.0)$ & \\
\hline$<2-5$ years & 16 & 80.0 & 4 & 20.0 & $20(100.0)$ & \\
\hline$<5-10$ years & 15 & 83.3 & 3 & 16.7 & $18(100.0)$ & \\
\hline More than 10 years & 25 & 89.3 & 3 & 10.7 & $28(100.0)$ & \\
\hline \multicolumn{6}{|c|}{ Attending training courses regarding gynecology and obstetrics } & \multirow{3}{*}{0.067} \\
\hline Yes & 30 & 85.7 & 5 & 14.3 & $35(100.0)$ & \\
\hline No & 38 & 74.5 & 13 & 25.5 & $51(100.0)$ & \\
\hline
\end{tabular}

(**) highly statistically significant $p<0.01$ 
Table (1): Shows personal data of maternity nurses, and reports that $67.4 \%$ them have age group between 20-30 years with a mean \pm SD of $28.45 \pm 2.71$, about $46.5 \%$ have a technical institute of nursing, $32.6 \%$ have more than 10 year of experience in their work, and about $59.3 \%$ attend training courses regarding gynecology and obstetrics .

Figure (1): Demonstrates that $52.3 \%$ of maternity nurses included in the study work at fetal monitoring and emergency ward, and about $24.4 \%$ of them work at intensive care ward.

Table (2): Shows knowledge of maternity nursing about APH in pre and posttest, and finds that there is highly statistical significance difference between pre \&posttest regarding definition, types, maternal and fetal complications p-value $(0.001,0.001,0.001$ and $0.002)$ respectively.

Table (3): Illustrates knowledge of maternity nursing about placenta previa in pre and posttest, and displays that there is highly statistical significance difference between pre \&posttest regarding general information, causes, and sign and symptoms of placenta previa $\mathrm{p}$ value 0.001 for all.

Table (4): Clarifies knowledge of maternity nursing about abruptio placenta in pre and posttest, and reports that there is highly statistical significance difference between pre \&posttest regarding general information, causes, and sign \& symptoms of abruptio placenta p-value $(0.002,0.001$, and 0.001$)$ respectively.

Table (5): Reveals nursing care of patient with APH in pre \& posttest, and illustrates that there is highly statistical significance difference between pre \&posttest regarding nursing care of APH p-value 0.001 and 0.033 .

Figure (2): Shows total knowledge of studied maternity nurses regarding APH in pre-test and posttest, and clarifies that there is highly statistical significance difference between pre $\&$ posttest pvalue 0.001 .

Table (6): Show that there is relation between total knowledge in pretest and age, educational qualification, and years of experience p-value 0.003, 0.001 and 0.002 respectively. And there is no relation between total knowledge in pretest and workplace and attending training courses p-value 0.119, and 0.678 .

Table (7): Demonstrates that there is relation between total knowledge in posttest and age, educational qualification, and years of experience $\mathrm{p}$ value $0.005,0.001$ and 0.003 respectively. And there is no relation between total knowledge in posttest and workplace and attending training courses p-value 0.495 , and 0.067 .

\section{Discussion}

Ante partum hemorrhage is a most serious obstetric problem and is a leading cause of maternal and fetal mortality and morbidity. It affects $2-5 \%$ of pregnancies. Nurses are the persons who play vital role in maintenance of health care as well as in the management of APH. Improving maternity nurse performance is extremely important and will be crucial in controlling blood loss and reducing the risk of maternal morbidity or even death (Emam, 2018). This study aims to assess maternity nurses' knowledge about APH., and evaluate effect of educational on improving maternity nurses' knowledge regarding APH.

According to total knowledge of studied maternity nurses regarding APH in pre-test and posttest, current study clarifies that there is highly statistical significance difference between pre \&posttest $\mathrm{p}$-value 0.001

This was on the same line with (Bishnoi, Ranjana, \& Geeyha, 2013), who applied their study in Bengaluru to assess the effectiveness of structure teaching program on knowledge regarding causes and intervention of APH among staff nurses, and found that there was a significance improvement of nurses knowledge regarding APH after implementation a teaching program.

Also (Emam, 2018), who achieved their study in Egypt to evaluate effect of an educational intervention on maternity nurses' performance regarding management of late $\mathrm{APH}$, and reported that there was highly statistical significance difference between pre \&posttest regarding knowledge about APH p-value 0.001.

Concerning general knowledge about APH, actual study demonstrates that there is statistical significance difference between pre \&posttest regarding definition, types, and complication of APH p-value 0.001, 0.001, and 0.002 .

This was similar to (Emam, 2018), who showed that there was statistical significance difference between pre \&posttest regarding definition, types, and complication of APH p-value 0.001 for all. Similarity between current study and (Bishnoi, Ranjana, \& Geeyha, 2013) \& (Emam, 2018) in knowledge about APH back to working on the same sample (maternity nurses).

Placenta previa is the most common cause of bleeding occurs in second and third trimesters of pregnancy. This condition occurs when the placenta implanted over or near the internal os of the cervix. Bleeding from it accounts for about $20 \%$ of all cases of APH (Hanon et al., 2012).

Regarding maternity nurses' knowledge about placenta previa as a main type of APH, present study reveals that there is statistical significance difference 
between pre \&posttest regarding general information, types, and causes, and signs and symptoms of placenta previa $p$-value 0.001 for all.

In agreement with previous result (Emam, 2018) clarifies that there was statistical significance difference between pre \&posttest regarding maternity nurses' knowledge about placenta previa.

Dissimilar to previous result (Hanon et al., 2012), who implemented their study in Baghdad, Iraq to evaluate the effect of educational program on pregnant women's knowledge about placenta previa, and reported that there was no statistical significance difference between pre \&posttest regarding signs and symptoms of placenta previa, dissimilarity between current study and (Hanon et al., 2012) back to difference in study sample.

Abruption Placenta is a premature partial or total separation of a normally situated placenta from the uterine wall before delivery of the fetal resulting in hemorrhage.it have three main types revealed, concealed, and combined (Riihimäki, 2018)

Current study shows that there is statistical significance difference between pre \&posttest regarding maternity nurses' knowledge about general information, types, causes, and signs and symptoms of abruption Placenta p-value $(0.002,0.001$, and 0.001 ) respectively.

This was on the same line with (Bishnoi, Ranjana, and Geeyha p, 2013), who reported that there was statistical significance difference between pre \&posttest regarding maternity nurses' knowledge about abruption Placenta.

When look to relationship between total knowledge about APH in pretest and maternity nurses' personal data, present study demonstrates that there is relation between total knowledge in pretest and age, educational qualification, and years of experience $\mathrm{p}$ value $0.003,0.001$ and 0.002 respectively. And there is no relation between total knowledge in pretest and workplace and attending training courses p-value 0.119 , and 0.678 .

Bishnoi, Ranjana, and Geeyha (2013), had the same view of opinion, who clarifies that there was relation between maternity nurses' knowledge and age, educational level, and years of experience p-value $<0.05$. Also (Mohammed \& Said, 2017), who carried out their study in Egypt to develop nursing management protocol for maternity nurses regarding obstetric emergencies, and displayed that there was relation between maternity nurses' knowledge and age and years of experience.

On the other side, (Elkholy et al., 2017), who applied their study in Egypt to assess nurses' practical skills regarding avoidance of postpartum hemorrhage, and illustrated that there was no relation between maternity nurses' knowledge and educational level, and years of experience. This may back to difference between current study and (Elkholy et al., 2017) in study subject.

Concerning personal data of studied maternity nurses, current study reports that more than two thirds of them have age group between 20-30 years with a mean \pm SD of $28.45 \pm 2.71$, less than half of them have a technical institute of nursing, around one third of studied nurses have more than 10 year of experience in their work, and nearly three fifths of them attend training courses regarding gynecology and obstetrics field.

Similar to previous results (Bishnoi, Ranjana, \& Geeyha, 2013) found that around two third of studied nurses had age of less than 35 years old, only one six of them have an educational level of bachelorate degree and less than one quarter work in intensive care unit.

Also (Mohammad \& Khaleel, 2019), who achieved their study in Iraq to assess midwives nurses' knowledge regarding care of postpartum hemorraghe, and reported that around one third of studied nurses had a ten years or more duration of experience and more than one third had a training course.

Disagreed with previous (Elkholy et al., 2017) showed that around one six of studied nurses had an age less than 30 years, three fifth of them had a secondary diploma of nursing, around two third had a ten years of experience in work and more than three quarters of them hadn't training course regarding obstetrics. This difference back to working in different setting.

\section{Conclusion}

There was an improvement in the knowledge of maternity nurses regarding APH evidenced by the pretest and posttest knowledge scores.

\section{Recommendations}

- Study should be generalized to include maternity nurses in both governmental and health hospital.

- Provide a continuous education and training programs to maternity nursing staff on $\mathrm{APH}$.

- Encourage nurses to update their education in the form of attending workshops, conferences related to care of APH.

- Supported maternity nurses with an instructional booklet regarding APH and its care to improve their knowledge and practices.

- Implement a study on effect of training program on maternity nurses' practice regarding care of APH patients.

\section{Acknowledgement}

The researchers would like to express their appreciation and thanks to all maternity nurses who involved in the study. 
References

- Ananth, C., Lavery, J., Vintzileos, A., Skupski, D., Varner, M., Saade, G., Biggio, J., Williams, M., Wapner, R., \& Wright, J., (2016): Severe placental abruption: Clinical definition and associations with maternal complications. American Journal of Obstetrics and Gynecology, 214(2), 272.e1-272.e9.

https://doi.org/10.1016/j.ajog.2015.09.069

- Bishnoi, Ranjana, Geeyha p., (2013): A study to assess the effectiveness of structure teaching program on knowledge regarding causes and intervention of APH among staff nurses in selected maternity hospitals at Bengaluru.

- Cresswell, J., Ronsmans, C., Calvert, C., \& Filippi, V., (2013): Prevalence of placenta praevia by world region: A systematic review and metaanalysis. Tropical Medicine and International Health, 18(6), 712-724. https://doi.org/10.1111/tmi.12100

- Debra Sullivan, S., on. (2016). Low-Lying Placenta (Placenta Previa), avialable at https://www.healthline.com/health/placenta-previa

- Elkholy, G., Ahmed, Abd Elsalsm Ramadan, S., Ouda, S., \& Abdel Fatah Ahmed, A., Abd elhamied. (2017): Assessment of Nurse's Practical Skills Regarding avoidance of Postpartum Hemorrhage. Egyptian Journal of Health Care, $8(3)$, 219-233. https://doi.org/10.21608/ejhc.2017.47309

- Emam, A., (2018): Maternity Nurses Performance Regarding Late Ante Partum Hemorrhage: An Educational Intervention. IOSR Journal of Nursing and Health Science (IOSR-JNHS), 7(4), 73-83. https://doi.org/10.9790/1959-0704107383

- Fan, D., Wu, S., Liu, L., Xia, Q., Wang, W., Guo, X., \& Liu, Z., (2017). Prevalence of antepartum hemorrhage in women with placenta previa: a systematic review and meta-analysis. Scientific reports, 7, 40320. https://doi.org/10.1038/srep40320

- Hanon, R., Al-saffar, F., \& Nursing, C., (2012): Assessment of Pregnant Women's Knowledge about Placenta Previa in Kirkuk Governorate Hospitals . R, Maternal and childhealth, 12(6).

- Jharaik, H., Dhiman, B., Verma, S., \& Sharma, A., (2019): Consequences of antepartum hemorrhage and its maternal and perinatal outcome. International Journal of Reproduction, Contraception, Obstetrics and Gynecology, 8(4), 1480 . 1770.ijrcog20191203

- Marianne Belleza, R., (2017): Placenta Previa Nursing Care Plan and Management. Https://Nurseslabs.Com/Placenta-Previa/.

- Mohammad, H., \& Khaleel, M., (2019): Assessment of nurse midwives knowledge regarding nursing care of post- partum hemorrhage at Al-Najaf Al-Ashraf city hospitals. Indian Journal of Public Health Research and Development, 10(10), 2952-2956. https://doi.org/10.5958/09765506.2019.03324.2

- Mohammed E., Abdelhakm \& Said A., Refaat (2017). Developing Nursing Management Protocol for Maternity Nurses Regarding Emergency Obstetric Care. American Journal of Nursing Science, $6(5), \quad 418$. https://doi.org/10.11648/j.ajns.20170605.16

- Norwitz, E., \& Shin Park, J., (2018): Overview of the etiology and evaluation of vaginal bleeding in pregnant women. Accessed via: http://www.uptodate.com /contents/overview-ofthe-etiology-and-evaluation-of vaginal-bleeding-in pregnant women? source $=$ machine Learning $\&$ search $=$ antepartum + haemorrhage $\&$ selected Title $=1 \% 7 \mathrm{E} 150 \&$ sectionRan $\mathrm{k}=3 \&$ anchor $=\mathrm{H} 28 \# \mathrm{H} 28$ $11 / 12 / 2018$

- Okby, R., Al Atawna, A., Wainstock, T., Sergienko, R., \& Sheiner, E., (2017): Placental abruption in twin pregnancies, risk factors and perinatal outcomes. American Journal of Obstetrics and Gynecology, 216(1), S551. https://doi.org/10.1016/j.ajog.2016.11.887

- Omokanye, L., Olatinwo, A., Salaudeen, A., Ajiboye, A., \& Durowade, K., (2017): A 5-year review of pattern of placenta previa in Ilorin, Nigeria. International Journal of Health Sciences, 11(2), 35-40. http://www.ncbi.nlm.nih.gov/pubmed/28539861\%0 Ahttp://www.pubmedcentral.nih.gov/articlerender.f cgi?artid=PMC5426413

- Rao, V., \& Hari, N., (2016): Maternal and perinatal outcome in abruptio placenta Study at teaching hospital. 3(10), 111-116.

- Riihimäki, O., (2018): placental Abruption LongTerm Morbidity and Mortality, Riihimäki, O. (2018). placental Abruption Long-Term Morbidity and Mortality. https://helda.helsinki.fi/bitstream/handle $/ 10138 / 253470$ /PLACENTA.pdf? sequence1\&isAllowedy.

- Sarojini Malini K. V., (2016): Clinical study of placenta previa and its effect on maternal health and fetal outcome. International Journal of Reproduction, Contraception, Obstetrics and Gynecology, 5(10), 3496-3499.

- Sharon Murray \& Emily McKinney (2017): foundation of maternal, newborn and women's health nursing, 6th edition, antepartum hemorrhage, p.p 72-99. 